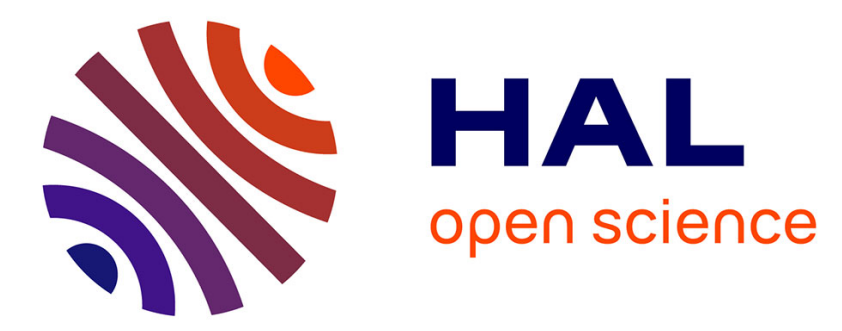

\title{
DNA-Controlled Spatiotemporal Patterning of a Cytoskeletal Active Gel
}

Yuliia Vyborna, Jean-Christophe Galas, André Estevez-Torres

\section{To cite this version:}

Yuliia Vyborna, Jean-Christophe Galas, André Estevez-Torres. DNA-Controlled Spatiotemporal Patterning of a Cytoskeletal Active Gel. Journal of the American Chemical Society, 2021, 10.1021/jacs.1c06730 . hal-03451234

HAL Id: hal-03451234

https://cnrs.hal.science/hal-03451234

Submitted on 26 Nov 2021

HAL is a multi-disciplinary open access archive for the deposit and dissemination of scientific research documents, whether they are published or not. The documents may come from teaching and research institutions in France or abroad, or from public or private research centers.
L'archive ouverte pluridisciplinaire HAL, est destinée au dépôt et à la diffusion de documents scientifiques de niveau recherche, publiés ou non, émanant des établissements d'enseignement et de recherche français ou étrangers, des laboratoires publics ou privés. 


\title{
DNA-controlled Spatio-temporal Patterning of a Cytoskele- tal Active Gel
}

\author{
Yuliia Vyborna*, Jean-Christophe Galas*, and André Estevez-Torres* \\ Sorbonne Université, CNRS, Institut de Biologie Paris-Seine (IBPS), Laboratoire Jean Perrin (LJP), F-75005, Paris, \\ France \\ KEYWORDS: Self-organization, DNA nanotechnology, active matter, DSD reaction.
}

\begin{abstract}
Living cells move and change their shape because signaling chemical reactions modify the state of their cytoskeleton; an active gel that converts chemical energy into mechanical forces. To create life-like materials, it is thus key to engineer chemical pathways that drive active gels. Here, we describe the preparation of DNA-responsive surfaces that control the activity of a cytoskeletal active gel comprised of microtubules: a DNA signal triggers the release of molecular motors from the surface into the gel bulk, generating forces that structure the gel. Depending on the DNA sequence and concentration, the gel forms a periodic band pattern or contracts globally. Finally, we show that the structuration of the active gel can be spatially controlled in the presence of a gradient of DNA concentration. We anticipate that such DNA-controlled active matter will contribute to the development of life-like materials with self-shaping properties.
\end{abstract}

Living cells and tissues change their shape autonomously because their cytoskeleton is an active gel that converts ATP chemical energy into mechanical work. ${ }^{1}$ To control their deformations during cell locomotion and development, cells and tissues tune the active state of their cytoskeleton over space and time. This is performed by chemical reactions that link the concentration of a trigger to the force exerted by the active gel. ${ }^{2}$ Despite its importance in the development of life-like synthetic materials, ${ }^{3,4}$ engineering such a chemo-mechanical trigger remains challenging. An important body of work has developed methods to chemically actuate hydrogels. ${ }^{5,6}$ However, current approaches either pattern passive hydrogels, ${ }^{7-10}$ or trigger deformations in active gels with chemical reactions that are difficult to design. ${ }^{11,12}$

A promising route relies on DNA hybridization reactions triggering forces that change the shape of hydrogels. Indeed, DNA reactions can be exquisitely controlled, ${ }^{13-17}$ and coupled to a great variety of materials. ${ }^{18-22}$ The mechanical amplification of a DNA signal can be either performed by DNA reactions, such as in DNA-responsive hydrogels, ${ }^{23,24}$ or by motor proteins, such as in DNA-based cytoskeletal active gels. ${ }^{25-29}$ While the former have the advantage of greater programmability, they lack the self-organization properties of cytoskeletal active gels. ${ }^{30-33}$ Despite progress in using DNA to tune the rheology of active gels ${ }^{29,34}$ or to trigger its activity at the micro-scale, ${ }^{25,26,35}$ we lack methods to control the spatio-temporal self-organization of cytoskeletal active gels at the macro-scale, as we demonstrate in the following.

The active gel used here is composed of clustered kinesin motors and microtubule filaments that are bundled together in the presence of a depletion agent. Because the filaments are continuously been propulsed by the motors such gels are known to self-organize into a variety of patterns. ${ }^{31,36-40}$ To control the activity of such gels with a DNA signal, kinesin clusters were attached to the DNA-modified surface of a glass channel filled with a solution of microtubule bundles (Fig. 1A). ${ }^{41,42}$ Motor clusters were released to the medium solely after the addition of DNA input I, thus initiating the continuous movement of microtubules and generating band structures followed by a global contraction of the gel (Fig. 1C). We demonstrate that the amplitude of these patterns is controlled by the sequence and the concentration of DNA. Furthermore, when the input DNA strand was heterogeneously distributed, it acted as a morphogen and provided a spatial control over the patterns observed in the active gel. Thus, this system paves the way toward the on-demand shaping of soft matter guided by DNA.

To prepare densely-covered DNA glass surfaces, acrylamide and acrydite-DNA $\mathbf{S}$ were copolymerized onto silanized glass slides (SI). ${ }^{43}$ These DNA-modified slides were further used to fabricate rectangular channels with typical dimensions of $22 \times 1.5 \times 0.1 \mathrm{~mm}^{3}$. Then, a solution of biotin-labeled target DNA T was added to the channel to form duplex S:T on the surface due to their partial complementarity (Fig. 1B). Subsequently, streptavidin (str) was injected into the channel and conjugated with biotinylated $\mathbf{T}$, generating complex S:T:str. Considering that $\mathbf{T}$ bears a single biotin and that streptavidin was added in 10-fold excess, the formation of 1:1 T:str complexes with three free biotin-binding sites in the streptavidin was expected. Finally, biotinylated kinesin (kin) was flown inside the 
channel to form S:T:str:kinn with a kin/str ratio, $\mathrm{n}$, that varied between 1 and 3 . This resulted in kinesin clusters attached to the surface and distributed homogeneously throughout the channel. Fluorescently labeled streptavidin was used to estimate the surface density of motor clusters that could be changed from $3 \times 10^{8}$ to $2 \times 10^{10}$ molecules $/ \mathrm{cm}^{2}$ (SI Fig. S1). The non-specific attachment of $\mathbf{T}$, str and kin to the surface was minimized in the presence of a $2 \%$ solution of Pluronic F-127 (SI Fig. S2).

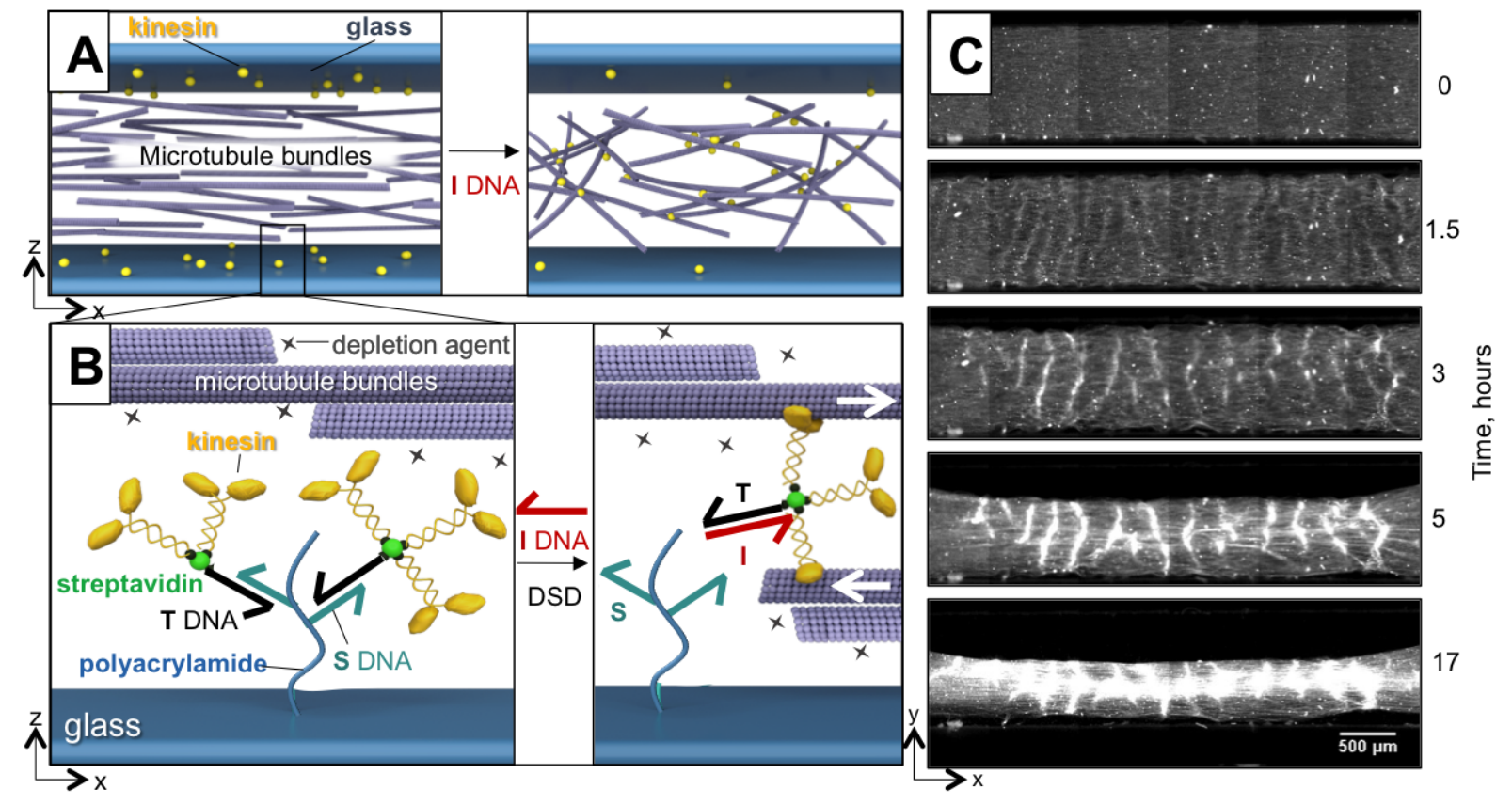

Figure 1. DNA-responsive, motor-decorated surfaces control the patterning of a cytoskeletal active gel. A: Schematic representation of a channel filled with microtubule bundles (noteworthy, the bundles are not polarized in contrast to polar microtubule filaments). Glass surfaces (in blue) are modified with kinesin-DNA constructs (yellow spheres). Upon addition of strand I, the motors are released into the bulk inducing forces upon the microtubules. B: Schematic representation of a glass surface functionalized by DNA/polyacrylamide brushes with kinesin-streptavidin-DNA constructs attached through DNA hybridized strands that undergo toehold-mediated strand displacement reaction. An invading strand I binds and displaces T DNA, releasing kinesin-streptavidinDNA constructs that bind to microtubules and move them relative to each other (white arrows). Microtubules form bundles due to the attraction interactions induced by depletion agent Pluronic (black stars). C: Time-lapse fluorescent microscopy images of the microtubule solution confined in glass channels in the presence of $1 \mu \mathrm{M}$ of I DNA. For detailed conditions see SI.

The kinesin-decorated channel was subsequently filled with a solution of microtubules (Fig. 1A) self-assembled into bundles in the presence of the depletion agent Pluronic. $^{31,44}$ The detachment of kinesin clusters from the surface was achieved through a DNA strand displacement (DSD) reaction in the presence of the invader strand $\mathbf{I}$ that is fully complementary to T (Fig. 1B). ${ }^{45}$ We thus have

$$
\text { S: T: str: } \operatorname{kin}_{\boldsymbol{n}}+\mathrm{I} \underset{k_{-1}}{\stackrel{k_{1}}{\rightleftharpoons}} \mathrm{S}+\mathrm{I}: \mathrm{T}: \mathbf{s t r}: \mathbf{k i n}_{\boldsymbol{n}}
$$

where the formation of the duplex I:T is thermodynamically favored compared to $\mathbf{S}: \mathbf{T}$ because the former is 5 base-pairs longer than the latter. Fig. 1C and Movie S1 show the behavior of the gel in the presence of $1 \mu \mathrm{M}$ of I. In the first 3 hours, we observed the formation of bright bands parallel to the $y$ axis with a typical period of $300 \pm$ $50 \mu \mathrm{m}$. These bands are reminiscent of the corrugations observed in a similar system where the kinesin clusters were initially present in solution. ${ }^{37,46}$ Afterwards, the gel contracted along $y$ and $x$, making the bands brighter. Noteworthy, no bands or contraction was observed in the absence of I or in the presence of a DNA strand with a random sequence (N) (Fig. S3 and Movie S1). The physical separation between the motors and the microtubules was key to obtain a robust inactive initial state. In a control experiment with kinesin clusters present in solution from the start, bands and contraction were also observed (Fig. S11). These observations are compatible with reaction (1), indicating that I releases kinesin clusters into the medium, inducing the mutual sliding of the bundles and shaping the active gel at the centimeter scale.

We measured the kinetics and thermodynamics of the forward reaction (1) by following the fluorescence from labeled-streptavidin on the glass surfaces. Fig. 2A shows that the characteristic time for releasing I:T:str:kin from the surface is equal to $5.5 \mathrm{~min}$ when $[\mathbf{I}]=1 \mu \mathrm{M}$ and thus the corresponding rate constant is $k_{1}=3 \times 10^{3} \mathrm{M}^{-1} \mathrm{~S}^{-1}$, which is compatible with previous measurements of dsDNA association kinetics on surfaces ${ }^{47}$ and $10^{3}$ times smaller than the typical value in solution. ${ }^{48}$ In the absence of $\mathbf{I}$, the streptavidin-associated fluorescence remained unchanged over time. To quantify active gel dynamics we defined a gel contraction index as the standard deviation of the microtubule intensity (see SI Section 6). The dynamics of the gel was significantly slower compared to the kinetics of the DNA reaction (Fig. 2B), with a characteristic time of 300 min. Fig. 2C (red) shows the titration of kinesins attached to the surface by increasing concentrations of I. We extracted an equilibrium constant $\mathrm{K}_{1}=2.3 \times 10^{-2}$ for reaction 
(1), similar to the one measured in the absence of microtubules $\left(\mathrm{K}^{*}{ }_{1}=3.6 \times 10^{-2}\right.$, SI Fig. S4) confirming that reaction (1) is driven by DNA hybridization and not by a potential kinesin-microtubule interaction. We estimated the corresponding equilibrium constant in solution at $22{ }^{\circ} \mathrm{C}$ to be 4 $\times 10^{6}$ (NUPACK) indicating that reaction (1) is significantly hindered on the surface compared to solution, as expected. ${ }^{49}$ Importantly, increasing [I] resulted in an increasingly contracted microtubule network (Fig 2C black) and we observed network structures from slightly contracted at $5 \mathrm{nM}$, to bright bands at $50 \mathrm{nM}$ and strong global contractions at $\geq 250 \mathrm{nM}$ (SI Fig. S7).

Changing the length of the duplex in the complex S:T, which allows attaching kinesin clusters to the surface, provides another way to control the activity of the gel via the DSD reaction. Indeed, shorter $\mathbf{S}: \mathbf{T}$ duplexes are expected to increase $\mathrm{K}_{1}$ and thus the final concentration of kinesin clusters in solution. In agreement with this interpretation, the amplitude of the gel deformations increased as the length of the duplex decreased at constant $[\mathbf{I}]=1 \mu \mathrm{M}$ (Fig. 2D and Movie S2). We observed faint regular bands for the $30 \mathrm{bp}$-long duplex, bright bands with local gel breaks for $22 \mathrm{bp}$, and bright bands with global contraction for the 15 bp-long duplex. Confocal images showed that faint bands correspond to a local increase in the microtubule density while bright bands were associated with gel corrugations in the $y z$ plane (Movie S4). A third parameter that
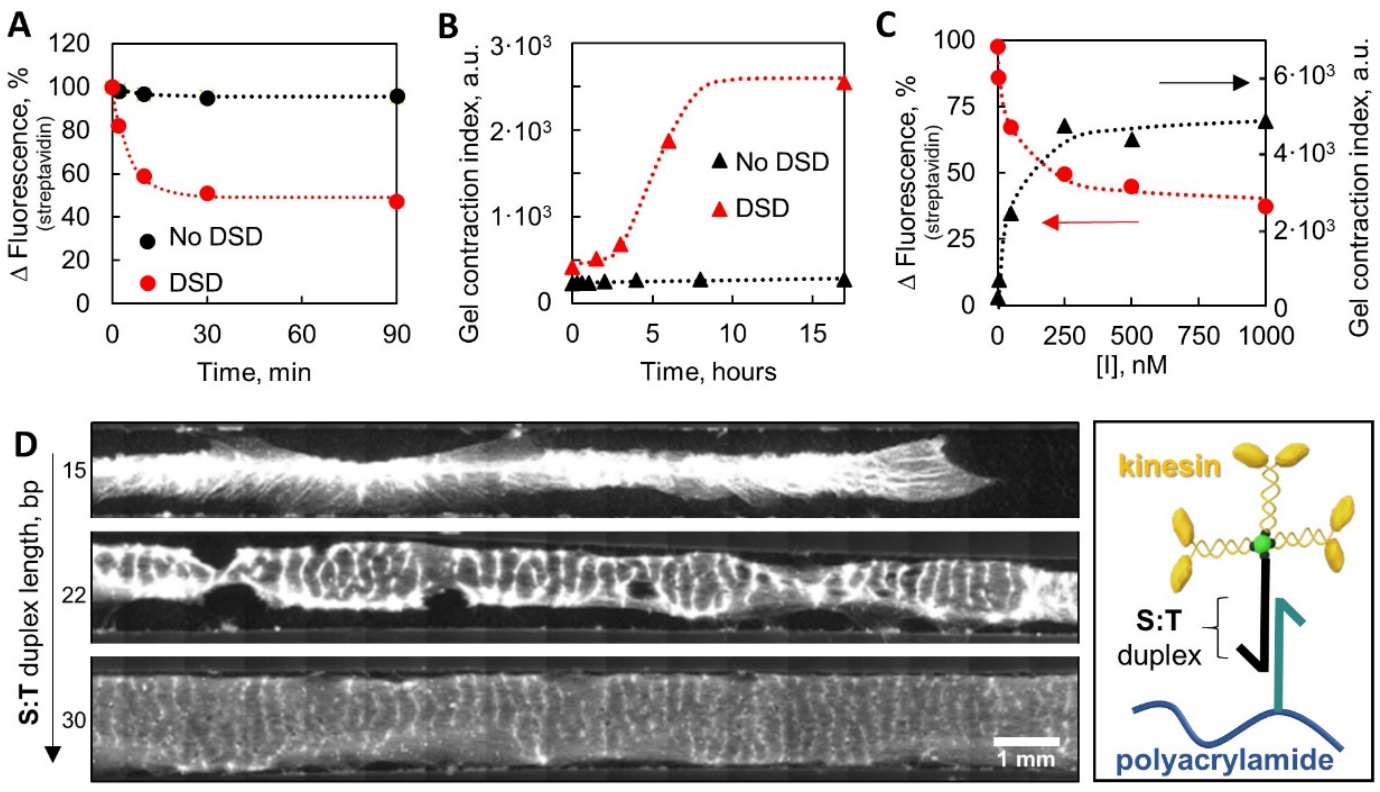

Figure 2: Influence of the kinetics and thermodynamics of the DSD reaction on the active gel patterning. Streptavidin fluorescence on the surface of the glass (A) and gel contraction index (B) as a function of time in the absence and in the presence of $1 \mu \mathrm{M}$ of $\mathbf{I}$. The dotted lines are fits to eqs. S2 and S8 respectively. Streptavidin fluorescence on the surface of the glass after 90 minutes (red) and gel contraction index after 17 hours (black) as a function of the concentration of I (C). The dotted lines are fits to eqs. S6 and S7. D: Fluorescence microscopy images of microtubules confined in glass channels modified with biotinylated DNA strands of different complementarity lengths in the presence of $1 \mu \mathrm{M}$ I strand after 18 hours. For detailed conditions see SI.

allows to control gel activity through DSD reaction is the concentration of the immobilized kinesin on the surface. Fig. S8 examines the effect of str surface density on the dynamics of the active gel for $[\mathbf{I}]=1 \mu \mathrm{M}$. For the densities of $0.5 \times, 1 \times$ and $2 \times 10^{10}$ molecules $/ \mathrm{cm}^{2}$ we respectively observed: slight bulk contraction with no bands, weak bands and bright bands with strong global contraction. Taken together, the data from Fig. 2, S3 and S7-S8 imply that the DSD reaction is fast compared to the dynamics of the active gel and that the state of the gel is determined by the concentration of motors released in solution, which is tuned by the thermodynamics of the DSD reaction and by the surface density of str.

In biology, morphogens play a crucial role in the patterning of developing tissues by diffusing in space and forming concentration gradients that provide positional information to downstream molecular events. In particular, morphogen gradients influence active matter processes in vivo. $^{50}$ In the following, we assessed the invader DNA strand as a morphogen that provides positional information to an active gel. To do so, $1 \mu \mathrm{M}$ of DNA I (the morphogen) was put solely on the left side of a channel filled with microtubules (Fig. 3 and SI Methods). On this side of the channel the onset of gel activity was detected after two hours followed by the formation of bright spots and global contraction in the $y$ direction. As expected, I diffused toward the right side forming a concentration gradient of $\mathbf{I}$, and thus of released kinesin clusters, that contracted the gel with decreasing amplitude and increased delay as the distance from the injection point increased (Fig. 3, Fig. S9 and Movie S3). In the central part of the channel, I arrived by diffusion at a concentration significantly lower than 1 $\mu \mathrm{M}$, and activity was observed after 10 hours with a front of activity progressing from left to right, with bright bands appearing sequentially. No activity was observed over the course of 43 hours in the right side of the channel where the morphogen concentration was negligible. 


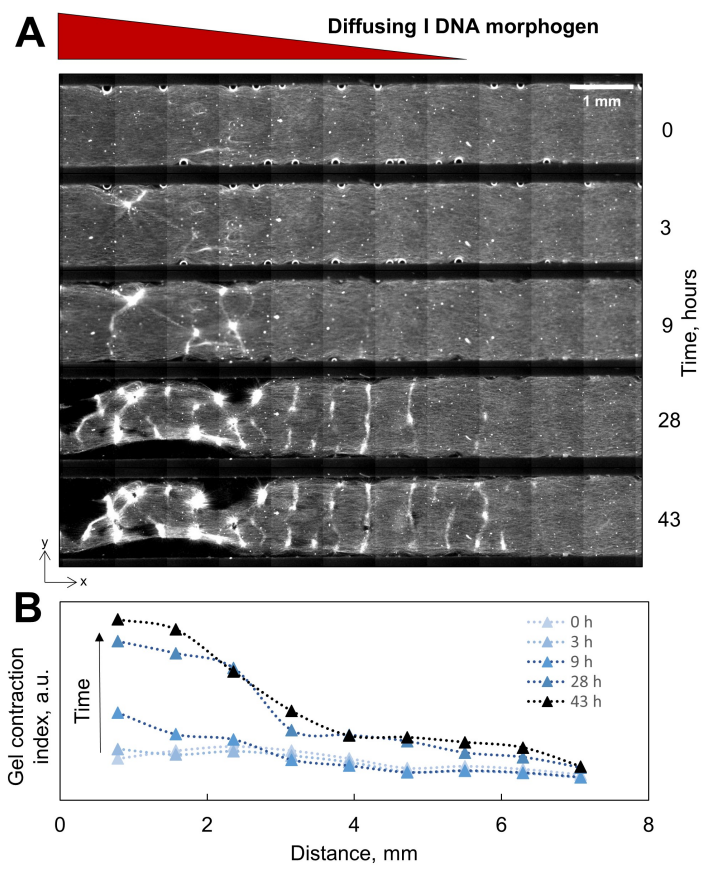

Figure 3: Active gel patterning through an underlying morphogen gradient of DNA. A: Time-lapse fluorescent microscopy images of the microtubule solution confined in glass channels after the addition of $1 \mu \mathrm{M}$ I DNA on the left side. For detailed conditions see SI. B: Gel contraction index computed along the channel length for each time point (Fig. S6). The dotted lines are guides to the eye.

The multiscale structuring of shapeless soft matter, including active materials, is conceptually and experimentally challenging. ${ }^{5}$ Here it was shown that the large-scale mechanical self-organization of microtubules could be activated in a programmable manner by a DNA signal. DNA-kinesin conjugates hybridized to a DNA-modified surface could be released in a controllable way by specific DNA inputs that trigger a DNA strand displacement reaction. Moreover, the DNA input could be distributed heterogeneously across space and act as a morphogen, transforming chemical information into a mechanical and structural output with spatial resolution. Complementary to the systems that utilize mechano-chemical transduction, ${ }^{51}$ this work provides an approach allowing for preparation of a synthetic material that actuates along a chemo-mechanical pathway. We anticipate that this methodology will open a new route to accessing novel self-fabricated, force-exerting synthetic soft matter with the potential of integration in soft robotics and biological environments.

\section{ASSOCIATED CONTENT}

DNA sequences, experimental details, including eqs S1-S8, additional Figures S1-S10, Movies S1-S4 and Tables S1 and S2. This material is available free of charge via the Internet at http://pubs.acs.org.

\section{AUTHOR INFORMATION}

\section{Corresponding Author}

Yuliia Vyborna - Sorbonne Université, F-75005 Paris,

France; orcid.org/0000-0003-0123-5971;
Email: yuliia.vyborna@sorbonne-universite.fr

Jean-Christophe Galas - Sorbonne Université, F-75005 Paris, France; Email: jean-christophe.galas@sorbonne-universite.fr André Estevez-Torres - Sorbonne Université, F-75005 Paris, France; orcid.org/0000-0002-5040-1369;

Email: andre.estevez-torres@sorbonne-universite.fr

\section{Author Contributions}

All authors contributed equally.

\section{Funding Sources}

We are thankful for the financial support from the European Research Council (ERC) under the European's Union Horizon 2020 program (grant no. 770940, A.E.-T.) and by a Marie Sklodowska-Curie fellowship (grant no. 888997, Y.V.) from the European Union's Horizon 2020 Program.

\section{REFERENCES}

(1) Prost, J.; Jülicher, F.; Joanny, J. Active Gel Physics. Nat. Phys. 2015, 11 (2), 111-117.

(2) Hannezo, E.; Heisenberg, C. P. Mechanochemical Feedback Loops in Development and Disease. Cell 2019, 178 (1), 1225.

Dogic, Z Active Matter at the Interface between Materials Science and Cell Biology. Nat. Rev. Mater. 2017, 2 (9), 17048.

Senoussi, A.; Vyborna, Y.; Berthoumieux, H.; Galas, J.-C.; Estevez-Torres, A. Learning from Embryo Development to Engineer Self-Organizing Materials. In Out-of-Equilibrium (Supra)molecular Systems and Materials; Giuseppone, N., Walther, A., Eds.; WILEY-VCH GmbH, 2021; pp 21-60.

(5) Chivers, P. R. A.; Smith, D. K. Shaping and Structuring Supramolecular Gels. Nat. Rev. Mater. 2019, 4 (7), 463-478.

(6) Ionov, L. Hydrogel-Based Actuators: Possibilities and Limitations. Biochem. Pharmacol. 2014, 17 (10), 494-503.

(7) Spitzer, D.; Marichez, V.; Formon, G. J. M.; Besenius, P.; Hermans, T. M. Surface-Assisted Self-Assembly of a Hydrogel by Proton Diffusion. Angew. Chemie Int. Ed. 2018, 57 (35), 11349-11353.

(8) Olive, A. G. L.; Abdullah, N. H.; Ziemecka, I.; Mendes, E. Eelkema, R.; van Esch, J. H. Spatial and Directional Control over Self-Assembly Using Catalytic Micropatterned Surfaces. Angew. Chemie 2014, 126 (16), 4216-4220.

(9) Lovrak, M.; Hendriksen, W. E. J.; Maity, C.; Mytnyk, S.; van Steijn, V.; Eelkema, R.; van Esch, J. H. Free-Standing Supramolecular Hydrogel Objects by Reaction-Diffusion. Nat Commun. 2017, 8 (1), 15317.

(10) Abe, K.; Murata, S.; Kawamata, I. Cascaded Pattern Formation in Hydrogel Medium Using the Polymerisation Approach Soft Matter 2021, 17 (25), 6160-6167.

(11) Yoshida, R.; Ueki, T. Evolution of Self-Oscillating Polymer Gels as Autonomous Polymer Systems. NPG Asia Mater 2014, 6 (6), e107.

(12) Li, Q.; Fuks, G.; Moulin, E.; Maaloum, M.; Rawiso, M.; Kulic, I Foy, J. T.; Giuseppone, N. Macroscopic Contraction of a Gel Induced by the Integrated Motion of Light-Driven Molecular Motors. Nat. Nanotechnol. 2015, 10 (2), 161-165.

(13) Montagne, K.; Plasson, R.; Sakai, Y.; Fujii, T.; Rondelez, Y. Programming an in Vitro DNA Oscillator Using a Molecular Networking Strategy. Mol. Syst. Biol. 2011, 7 (1), 466.

(14) Padirac, A.; Fujii, T.; Estévez-Torres, A.; Rondelez, Y. Spatial Waves in Synthetic Biochemical Networks. J. Am. Chem. Soc. 2013, 135 (39), 14586-14592.

(15) Heinen, L.; Walther, A. Programmable Dynamic Steady States in ATP-Driven Nonequilibrium DNA Systems. Sci. Adv. 2019, 5 (7), 32-37.

(16) Zhang, D. Y.; Seelig, G. Dynamic DNA Nanotechnology Using Strand-Displacement Reactions. Nat. Chem. 2011, 3 (2), 103-113

(17) Yurke, B.; Turberfield, A. J.; Mills, A. P.; Simmel, F. C.; Neumann, J. L. A DNA-Fuelled Molecular Machine Made of DNA. Nature 2000, 406 (6796), 605-608. 
(18) He, L.; Mu, J.; Gang, O.; Chen, X. Rationally Programming Nanomaterials with DNA for Biomedical Applications. Adv. Sci. 2021, 8 (8), 2003775.

(19) Vyborna, Y.; Vybornyi, M.; Häner, R. From Ribbons to Networks: Hierarchical Organization of DNA-Grafted Supramolecular Polymers. J. Am. Chem. Soc. 2015, 137 (44), 14051-14054

(20) Fern, J.; Schulman, R. Modular DNA Strand-Displacement Controllers for Directing Material Expansion. Nat. Commun. 2018, 9 (1), 3766.

(21) Buchberger, A.; Simmons, C. R.; Fahmi, N. E.; Freeman, R.; Stephanopoulos, N. Hierarchical Assembly of Nucleic Acid/Coiled-Coil Peptide Nanostructures. J. Am. Chem. Soc. 2020, 142 (3), 1406-1416.

(22) Zadorin, A. S.; Rondelez, Y.; Gines, G.; Dilhas, V.; Urtel, G.; Zambrano, A.; Galas, J.-C.; Estevez-Torres, A. Synthesis and Materialization of a Reaction-Diffusion French Flag Pattern. Nat. Chem. 2017, 9 (10), 990-996.

(23) Jeon, S.-J.; Hauser, A. W.; Hayward, R. C. Shape-Morphing Materials from Stimuli-Responsive Hydrogel Hybrids. Acc. Chem. Res. 2017, 50 (2), 161-169.

(24) Cangialosi, A.; Yoon, C.; Liu, J.; Huang, Q.; Guo, J.; Nguyen, T. D.; Gracias, D. H.; Schulman, R. DNA Sequence-Directed Shape Change of Photopatterned Hydrogels via High-Degree Swelling. Science (80-. ). 2017, 357 (6356), 1126-1130.

(25) Wollman, A. J. M.; Sanchez-Cano, C.; Carstairs, H. M. J.; Cross, R. A.; Turberfield, A. J. Transport and Self-Organization across Different Length Scales Powered by Motor Proteins and Programmed by DNA. Nat. Nanotechnol. 2014, 9 (1), 4447.

(26) Keya, J. J.; Suzuki, R.; Kabir, A. M. R.; Inoue, D.; Asanuma, H.; Sada, K.; Hess, H.; Kuzuya, A.; Kakugo, A. DNA-Assisted Swarm Control in a Biomolecular Motor System. Nat. Commun. 2018, 9 (1), 453.

(27) Sato, Y.; Hiratsuka, Y.; Kawamata, I.; Murata, S.; Nomura, S. M. Micrometer-Sized Molecular Robot Changes Its Shape in Response to Signal Molecules. Sci. Robot. 2017, 2 (4), eaal3735.

(28) Matsuda, K.; Kabir, A. M. R.; Akamatsu, N.; Saito, A.; Ishikawa, S.; Matsuyama, T.; Ditzer, O.; Islam, M. S.; Ohya, Y.; Sada, K.; Konagaya, A.; Kuzuya, A.; Kakugo, A. Artificial Smooth Muscle Model Composed of Hierarchically Ordered Microtubule Asters Mediated by DNA Origami Nanostructures. Nano Lett. 2019, 19 (6), 3933-3938.

(29) Tayar, A. M.; Hagan, M. F.; Dogic, Z. Active Liquid Crystals Powered by Force-Sensing DNA-Motor Clusters. Proc. Natl. Acad. Sci. 2021, 118 (30), e2102873118.

(30) Nédélec, F. J.; Surrey, T.; Maggs, A. C.; Leibler, S. SelfOrganization of Microtubules and Motors. Nature 1997, 389 (6648), 305-308.

(31) Sanchez, T.; Chen, D. T. N.; DeCamp, S. J.; Heymann, M.; Dogic, Z. Spontaneous Motion in Hierarchically Assembled Active Matter. Nature 2012, 491 (7424), 431-434.

(32) Popkin, G. The Physics of Life. Nature 2016, 529 (7584), 1618.

(33) Duclos, G.; Adkins, R.; Banerjee, D.; Peterson, M. S. E.; Varghese, M.; Kolvin, I.; Baskaran, A.; Pelcovits, R. A.; Powers, T. R.; Baskaran, A.; Toschi, F.; Hagan, M. F.; Streichan, S. J.; Vitelli, V.; Beller, D. A.; Dogic, Z. Topological Structure and Dynamics of Three-Dimensional Active Nematics. Science (80-. ). 2020, 367 (6482), 1120-1124.

(34) Matsuda, K.; Kabir, A. M. R.; Akamatsu, N.; Saito, A.; Ishikawa, S.; Matsuyama, T.; Ditzer, O.; Islam, M. S.; Ohya, Y.; Sada, K.; Konagaya, A.; Kuzuya, A.; Kakugo, A. Artificial Smooth Muscle Model Composed of Hierarchically Ordered Microtubule Asters Mediated by DNA Origami Nanostructures. Nano Lett.
2019, 19 (6), 3933-3938.

(35) Sato, Y.; Hiratsuka, Y.; Kawamata, I.; Murata, S.; Nomura, S. I. M. SI Micrometer-Sized Molecular Robot Changes Its Shape in Response to Signal Molecules. Sci. Robot. 2017, 2 (4).

(36) Wu, K.-T.; Hishamunda, J. B.; Chen, D. T. N.; DeCamp, S. J.; Chang, Y.-W.; Fernández-Nieves, A.; Fraden, S.; Dogic, Z Transition from Turbulent to Coherent Flows in Confined Three-Dimensional Active Fluids. Science (80-. ). 2017, 355 (6331), eaal1979.

(37) Senoussi, A.; Kashida, S.; Voituriez, R.; Galas, J.-C.; Maitra, A.; Estevez-Torres, A. Tunable Corrugated Patterns in an Active Nematic Sheet. Proc. Natl. Acad. Sci. 2019, 116 (45), 22464 22470 .

(38) Lemma, B.; Mitchell, N. P.; Subramanian, R.; Needleman, D. J.; Dogic, Z. Active Microphase Separation in Mixtures of Microtubules and Tip-Accumulating Molecular Motors. 2021, arXiv:2107.12281v2.

(39) Torisawa, T.; Taniguchi, D.; Ishihara, S.; Oiwa, K. Spontaneous Formation of a Globally Connected Contractile Network in a Microtubule-Motor System. Biophys. J. 2016, $111(2), 373-385$.

(40) Nitta, T.; Wang, Y.; Du, Z.; Morishima, K.; Hiratsuka, Y. A Printable Active Network Actuator Built from an Engineered Biomolecular Motor. Nat. Mater. 2021, 20 (8), 1149-1155.

(41) Niemeyer, C. M.; Sano, T.; Smith, C. L.; Cantor, C. R. Oligonucleotide-Directed Self-Assembly of Proteins: Semisynthetic DNA-Streptavidin Hybrid Molecules as Connectors for the Generation of Macroscopic Arrays and the Construction of Supramolecular Bioconjugates. Nucleic Acids Res. 1994, 22 (25), 5530-5539.

(42) Hager, R.; Arnold, A.; Sevcsik, E.; Schütz, G. J.; Howorka, S. Tunable DNA Hybridization Enables Spatially and Temporally Controlled Surface-Anchoring of Biomolecular Cargo. Langmuir 2018, 34 (49), 15021-15027.

(43) Rehman, F. N.; Audeh, M.; Abrams, E. S.; Hammond, P. W.; Kenney, M.; Boles, T. C. Immobilization of AcrylamideModified Oligonucleotides by Co-Polymerization. Nucleic Acids Res. 1999, 27 (2), 649-655.

(44) Braun, M.; Lansky, Z.; Hilitski, F.; Dogic, Z.; Diez, S. Entropic Forces Drive Contraction of Cytoskeletal Networks. BioEssays 2016, 38 (5), 474-481.

(45) Simmel, F. C.; Yurke, B.; Singh, H. R. Principles and Applications of Nucleic Acid Strand Displacement Reactions. Chem. Rev. 2019, 119 (10), 6326-6369.

(46) Strübing, T.; Khosravanizadeh, A.; Vilfan, A.; Bodenschatz, E.; Golestanian, R.; Guido, I. Wrinkling Instability in 3D Active Nematics. Nano Lett. 2020, 20 (9), 6281-6288.

(47) Gao, Y. Secondary Structure Effects on DNA Hybridization Kinetics: A Solution versus Surface Comparison. Nucleic Acids Res. 2006, 34 (11), 3370-3377.

(48) Morrison, L. E.; Stols, L. M. Sensitive Fluorescence-Based Thermodynamic and Kinetic Measurements of DNA Hybridization in Solution. Biochemistry 1993, 32 (12), 30953104.

(49) Irving, D.; Gong, P.; Levicky, R. DNA Surface Hybridization: Comparison of Theory and Experiment. J. Phys. Chem. B 2010, 114, 7631-7640.

(50) Collinet, C.; Lecuit, T. Programmed and Self-Organized Flow of Information during Morphogenesis. Nat. Rev. Mol. Cell Biol. 2021, 22 (4), 245-265.

(51) Senoussi, A.; Galas, J.-C.; Estevez-Torres, A. Programmed Mechano-Chemical Coupling in Reaction-Diffusion Active Matter. bioRxiv 2021, 2021.03.13.435232. 


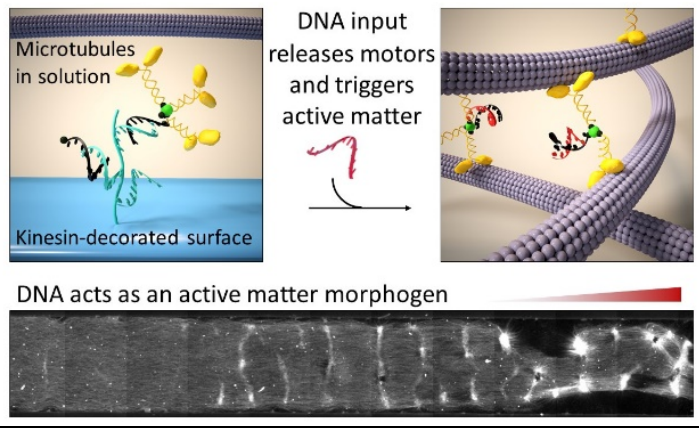

